\title{
A Specific Inhibitory Pathway between Substantia Gelatinosa Neurons Receiving Direct C-Fiber Input
}

\author{
Yan Lu and Edward R. Perl \\ Department of Cell and Molecular Physiology, University of North Carolina, Chapel Hill, North Carolina 27599
}

\begin{abstract}
The spinal substantia gelatinosa (SG) is a major termination region for unmyelinated (C) primary afferent fibers; however, how the input it receives from these sensory fibers is processed by SG neurons remains primarily a matter of conjecture. To gain insight on connections and functional interactions between intrinsic SG neurons, simultaneous tight-seal, whole-cell recordings were made from pairs of neurons in rat spinal cord slices to examine whether impulses in one cell generated synaptic activity in the other. Most SG neuron pairs sampled lacked synaptic interaction. Those showing a linkage included a recurring pattern consisting of a monosynaptic, bicucullinesensitive inhibitory connection from an islet cell to a transient central neuron, each of which received direct excitatory input from different afferent C-fibers. This newly defined inhibitory circuit is postulated to represent a SG neural module by which a nociceptive C-fiber input to transient central cells is modified by other C-fiber messages.
\end{abstract}

Key words: inhibitory pathway; substantia gelatinosa; spinal lamina II; afferent C-fibers; islet neurons; $\mathrm{GABA}_{\mathrm{A}}$; nociception

\section{Introduction}

The substantia gelatinosa (SG, lamina II) of the superficial dorsal horn of the spinal cord receives many synaptic terminations from primary afferent neurons with unmyelinated $(\mathrm{C})$ and thinly myelinated (A $\delta$ ) fibers (Perl, 1984; Light, 1992). Because much of the afferent signaling by such thin fibers is related to nociception and pain, it is widely presumed that the SG plays an important part in pain mechanisms(Willis and Coggeshall, 1978; Perl, 1984; Light, 1992). The fact that some $\mathrm{A} \delta$ and C primary afferent neurons projecting to the SG convey information about innocuous thermal and mechanical events (Perl, 1984; Sugiura et al., 1986; Light, 1992) is often overlooked.

The SG is a complex neuropil characterized by a paucity of myelinated fibers. Neurons with somata in the SG represent a variety of cellular configurations and neurite distributions (Ramon y Cajal, 1909; Pearson, 1952; Gobel, 1975, 1978; Beal et al., 1989; Grudt and Perl, 2002). Lamina II also contains a rich and diverse concentration of molecules that act as synaptic mediators or are substances that influence neuronal excitability (for review, see Perl, 1984; Light, 1992; Budai, 2000). A number of studies have reported on the primary afferent input of the SG and responses of SG neurons to such input (Szentágothai, 1964; Réthelyi and Szentágothai, 1969; Gobel, 1975; Kumazawa and Perl, 1978; Light et al., 1979; Cervero and Iggo, 1980; Gobel et al., 1980); however, there is a remarkable lack of information on the

Received June 10, 2003; revised July 31, 2003; accepted Aug. 1, 2003.

This work was supported by Research Grant NS-10321 from the National Institute of Neurological Disorders and Stroke-National Institutes of Health. We are grateful to Adam Hantman for valuable help with the confocal microscope. Kirk McNaughton provided excellent histological assistance. We thank Sherry Joseph for substantial aid in preparation of this manuscript.

Correspondence should be addressed to Edward R. Perl, Department of Cell and Molecular Physiology, University of North Carolina, 5109 Neuroscience Research Building, Campus Box 7545, Chapel Hill, NC 27599. E-mail: erp@med.unc.edu.

Copyright $\odot 2003$ Society for Neuroscience $\quad$ 0270-6474/03/238752-07\$15.00/0 intrinsic circuitry of this region and how neuronal connections and the complex of molecular signals interact. As a step toward providing such data, simultaneous whole-cell recordings from two SG neurons were made to establish synaptic linkages between identified neurons. Herein, we provide an initial description of an inhibitory connection between specific intrinsic neurons of the $\mathrm{SG}$ that receive direct input from afferent C-fibers.

\section{Materials and Methods}

All procedures on living animals were approved by the Institutional Animal Care and Use Committee of the University of North Carolina Chapel Hill and conform to guidelines suggested by the Institute of Laboratory Animal Resources, Commission on Life Sciences (1996). Young adult Sprague Dawley rats of both sexes (6-10 weeks old) were deeply anesthetized with urethane $(1.5 \mathrm{gm} / \mathrm{kg}$, i.p.), and lumbosacral laminectomies were performed in a cold room. The lumbosacral spinal cord with dorsal roots was quickly removed and placed in ice-cold, sucrosesubstituted artificial CSF (sucrose ACSF, in mM: sucrose, 234; KCl, 3.6; $\mathrm{CaCl}_{2}, 2.5 ; \mathrm{MgCl}_{2}, 1.2 ; \mathrm{NaH}_{2} \mathrm{PO}_{4}, 1.2 ; \mathrm{NaHCO}_{3}, 25$; and D-glucose, 12). The rat was killed with an overdose of pentobarbital. Transverse (600$800 \mu \mathrm{m})$ or sagittal $(400-600 \mu \mathrm{m})$ lumbar spinal cord slices with attached dorsal roots were prepared in ice-cold sucrose ACSF using a vibrating microtome. The slices were then maintained and studied at room temperature $\left(22-25^{\circ} \mathrm{C}\right)$ in regular ACSF equilibrated with $95 \% \mathrm{O}_{2}$ and $5 \% \mathrm{CO}_{2}$ (ACSF, in mM: $\mathrm{NaCl}, 125 ; \mathrm{KCl}, 2.5 ; \mathrm{CaCl}_{2}, 2 ; \mathrm{MgCl}_{2}, 1$, $\mathrm{NaH}_{2} \mathrm{PO}_{4}, 1.25 ; \mathrm{NaHCO}_{3}, 26$; and D-glucose, 25).

A spinal cord slice was mounted in a small $(\sim 300 \mu \mathrm{l})$ superfusion chamber (ACSF flow of 4-6 $\mathrm{ml} / \mathrm{min}$ ) on a fixed stage, upright compound microscope (E600FM; Nikon, Mellville, NY) fitted with longworking distance, water immersion objectives and illuminated by infrared radiation $(790-810 \mathrm{~nm})$. Images of the slice were captured by a CCD video camera and displayed on a video monitor. Patch-type pipette electrodes with resistances of 5-10 $\mathrm{M} \Omega$ were filled with a solution containing (in $\mathrm{mM}$ ): $\mathrm{K}$ gluconate, $130 ; \mathrm{KCl}, 5 ; \mathrm{Mg}$-ATP, 4 ; phosphocreatine, 10; Li-GTP, 0.3; and HEPES, 10, pH 7.3, 300 mOsm. Biocytin (0.5\%) was back-filled in the recording pipettes. Additional details of the slice preparation and recording techniques are provided in previous publications 
(Yoshimura and Jessell, 1989; Li and Perl, 1994; Grudt and Perl, 2002; Grudt et al., 2002).

After one tight-seal, whole-cell recording was obtained from an SG neuron, another pipette electrode was brought in from the opposite side to make a second whole-cell recording from a nearby $(10-250 \mu \mathrm{m}) \mathrm{SG}$ neuron (Fig. 1). Recordings were considered to be from "healthy" neurons if stable transmembrane potentials were more negative than -45 $\mathrm{mV}$, and overshooting action potentials were generated by an inward (depolarizing) current introduced through the recording electrode. In voltage-clamp mode, the whole-cell recordings usually exhibited spontaneous, irregularly appearing, small, transient inward and outward postsynaptic currents. Series resistances typically were $\sim 10 \mathrm{M} \Omega$ and were monitored throughout the recording period. Recording from a cell was discarded if the series resistance changed significantly $( \pm 25 \%)$ and the original value could not be recovered by manipulation of suction applied to the recording pipette. For reasons of recording stability, series resistance compensation was not used. Because series resistance was at least an order of magnitude less than the cell input resistances, voltage or current measurement errors attributable to series resistance were presumed to be $<10 \%$.

Graded 0.1-0.5 msec pulses delivered through a suction electrode were used to initiate afferent volleys in the segmental dorsal root (DR). Conduction velocities of primary afferent fibers evoking monosynaptic EPSPs (or EPSCs under voltage clamp) were estimated from the latency of the evoked response and the conduction distance. A dorsal root evoked response was judged to be monosynaptic if it had a constant latency in repetitive trials (Yoshimura and Nishi, 1993; Li and Perl, 1994). Each neuron of a pair was stimulated in turn with $10 \mathrm{msec}$ rectangular $0.1-20 \mathrm{~Hz}$ current pulses, suprathreshold for generation of action potentials, to test for synaptic connections. The putative postsynaptic neuron was examined at its resting potential and in a partially depolarized state $(-35 \mathrm{mV})$. In the connected pairs, the latency of an EPSP (recorded at $-60 \mathrm{mV}$ ) or an IPSP (recorded at $-50 \mathrm{mV}$ ) was measured from peak of the presynaptic action potential. To characterize neurons, the firing pattern in current clamp was determined to a $1 \mathrm{sec}$ depolarizing pulse at a holding potential of $-60 \mathrm{mV}$.

Only one pair of neurons was studied in a slice. Very careful note was made of the position of each recording pipette relative to the other and to landmarks of the slice. Neurons were considered to be well stained by biocytin if the dendrite-like processes appeared to taper and terminated smoothly, and the arborization pattern lacked obvious deficits (Grudt and Perl, 2002).

Data acquisition and analysis were conducted using Axopatch 200B amplifiers (Axon Instruments), a Digidata 1200A interface, and pCLAMP 6 and 8 software (Axon Instruments). After the electrophysiological recordings, spinal slices were fixed by immersion in cold $4 \%$ paraformaldehyde for 3-8 d. Transverse slices were sectioned parasagittally at $60 \mu \mathrm{m}$ in a cryostat. Sagittal slices were processed whole. The biocytin label was visualized by the avidin-biotin complex technique (ABC kit; Vector Laboratories, Burlingame, CA). Images of the labeled neurons were captured using a compound microscope fitted with a digital camera (DEI-470; Optronics) or by a confocal microscope (Leica DMB IRBE). Many of the recordings were done in sagittally cut slices. In sagittal preparations, the dendritic extent was measured in the whole mounts. In recordings from transverse slices, the mediolateral dendritic extent was calculated from the number of serial $60 \mu \mathrm{m}$ sections involved. Measurements from sagittal whole mounts were considered the more accurate for rostrocaudal dimensions. Digital images were reconstructed in three dimensions using Neurolucida software (MicroBrightfield) with a Windows-based digital computer. Differences between rostrocaudal, mediolateral, and dorsoventral dendritic arbors were consistent and were judged to reflect neuronal geometry rather than orientation of the histological material. Data are presented as mean \pm SD. Two-sample Student's $t$ test was used to estimate the possibility of chance differences in values ( $p<0.05$ was taken as significant).

Chemical compounds and pharmacological agents [6-cyano-7 nitroquinoxaline-2,3 dione (CNQX), bicuculline, and strychnine] were obtained from Sigma (St. Louis, MO).

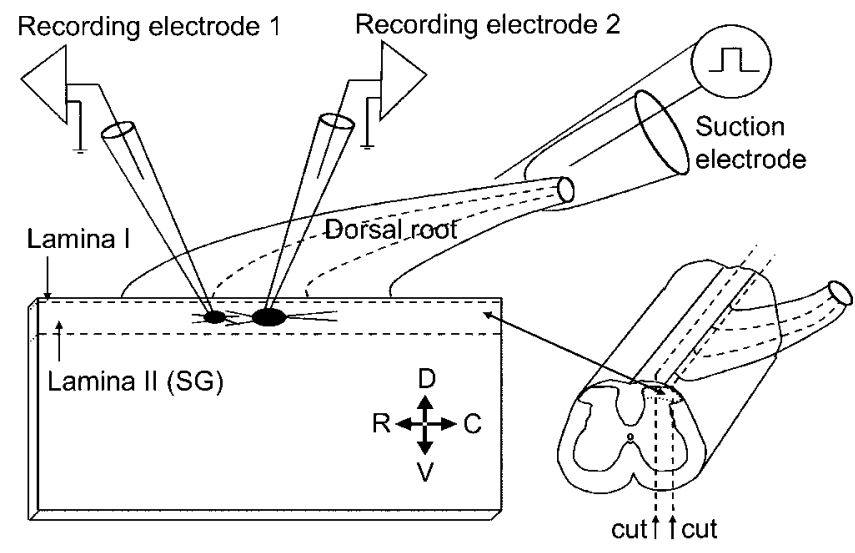

Figure 1. Diagram of the typical experimental arrangement. Sagittal spinal slices (400-600 $\mu \mathrm{m}$ thick, 3-5 mm long) with an attached dorsal root (8-10 $\mathrm{mm}$ long) were cut from the lumber spinal cord of adult Sprague Dawley rats and maintained in a recording chamber superfused with ACSF. Separate recording electrodes were used to make simultaneous tight-seal, whole-cell recordings from two SG neurons. A suction electrode was used to stimulate a segmental dorsal root to evoke volleys of impulses in primary afferent fibers. C, Caudal; $D$, dorsal; $R$, rostral; V, ventral.

\section{Results}

In the experimental arrangement shown in the diagram in Figure 1 , independently manipulating two patch-type electrodes under infrared guidance regularly yielded simultaneous tight-seal, whole-cell recordings from pairs of rat lamina II neurons. In the course of these experiments, electrophysiological observations were made on $>450$ individual SG neurons. Data from the 146 analyzed in detail morphologically generally confirm descriptions and the classification developed from similar information obtained in a systematic survey of hamster SG neurons (Grudt and Perl, 2002). In particular, it was readily possible to distinguish rat SG neurons that correspond to islet (36 of 146), tonic central (12 of 146), transient central (39 of 146), vertical (41 of 146 ), and radial (18 of 146) cell types on the grounds of criteria established in the hamster study. Although the present study was not designed as a broad survey, these similarities led us to use the hamster classification as a foundation for the present analysis.

Here we report evidence for prompt synaptic linkages between pairs of neurons, indicated by a consistent temporal relationship between action potentials evoked in one cell and subsequent postsynaptic potentials (or currents) in the other. When an action potential in one neuron was linked in time to a postsynaptic change in the other cell, special note was made of successive time differences between the two events. Small $(<0.2 \mathrm{msec})$ variations between the peak of the presynaptic action potential and the beginning of the evoked postsynaptic activity at various repetition rates were taken to indicate that the connection between the two neurons was monosynaptic (Yoshimura and Jessell, 1989; Li and Perl, 1994).

On the basis of these considerations, synaptic connections between randomly chosen SG neurons were infrequent. Our search concentrated on cells with somata separated by $<250 \mu \mathrm{m}$ in the center of the SG, near the interface of laminae IIi and IIo. Twenty-eight of 248 neuron pairs (11\%) evidenced synaptic interaction. Connected pairs were found more commonly in sagittal than transverse spinal cord slices, probably reflecting the dominant orientation of neurites of mid SG neurons in the rostrocaudal dimension. All of the synaptic connections between SG neurons were unidirectional; that is, activating the "postsynaptic" cell by depolarizing current never produced evidence of a 

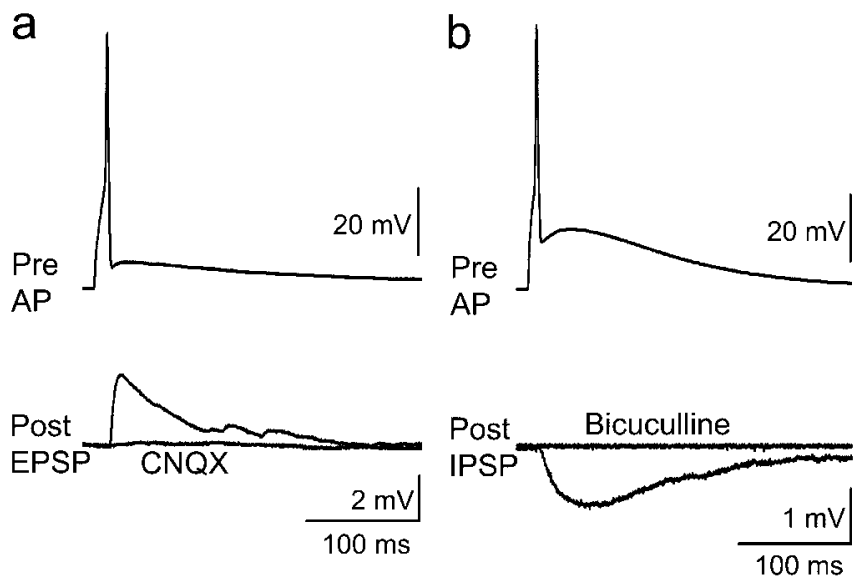

Figure 2. Simultaneous whole-cell recordings from two pairs of synaptically coupled SG neurons. $a$, Top trace, Current-clamp recording from the presynaptic (Pre) neuron of one pair (membrane potential, $-60 \mathrm{mV}$ ). An action potential (AP) was initiated by brief depolarizing current injection (10 msec, $1 \mathrm{nA}$ ) through the recording electrode. Bottom trace, Current-clamp recording from the postsynaptic (Post) neuron (membrane potential, $-60 \mathrm{mV}$ ) showing an excitatory postsynaptic potential (Post EPSP) evoked by the presynaptic AP and its block by bath-applied CNQX (20 $\mu \mathrm{m})$. b, Top trace, Current-clamp recording from the presynaptic neuron of another pair of neurons (membrane potential, $-60 \mathrm{mV}$ ) showing an AP evoked by a brief current injection (10 msec, $1 \mathrm{nA}$ ) through the recording electrode. Bottom trace, Current-clamp recording from the postsynaptic neuron of this pair (membrane potential, $-50 \mathrm{mV}$ ) showing an inhibitory postsynaptic potential (Post IPSP) evoked by the presynaptic AP and its suppression by bath-applied bicuculline $(10 \mu \mathrm{M}), \mathrm{a} G \mathrm{GBA}_{\mathrm{A}}$ receptor antagonist. All traces are the average of $40-50$ individual records.

reciprocal synaptic connection to the original "presynaptic" neuron.

Three types of connections were noted. First, an action potential in one cell (presynaptic) generated an EPSP or EPSC in the other neuron (Fig. $2 a$ ) in 13 of 28 connected pairs. Small variations in latency of the evoked postsynaptic response on successive presynaptic action potentials suggested that the connection was monosynaptic in 12 of the 13 pairs. Although stable in latency, these evoked EPSPs were small, typically $<4 \mathrm{mV}$. Excitatory linkages were regularly blocked (Fig. $2 a$ ) by the glutamate AMPA receptor antagonist CNQX $(10 \mu \mathrm{M})$ in the superfusing ACSF. Second, in one case, the latency between the presynaptic action potential in one cell and the EPSP in the other neuron varied substantially ( $>1 \mathrm{msec}$ ); this was presumed to reflect a polysynaptic pathway between the two neurons. Third, for the majority of connected pairs (15 of 28), a presynaptic action potential was followed by a postsynaptic hyperpolarizing (inhibitory) potential (Fig. 2b). In all but one inhibitory connection, the evoked IPSP developed relatively slowly (time to peak, $\sim 35 \mathrm{msec}$ ) and was prolonged ( $50 \%$ decay, $\sim 98 \mathrm{msec}$ ). These prolonged IPSPs were reversibly eliminated by the $\mathrm{GABA}_{\mathrm{A}}$ receptor antagonist bicuculline $(10 \mu \mathrm{M} ; n=9)$, as illustrated in Figure $2 b$, and were unaffected by the glycine receptor antagonist strychnine $(1 \mu \mathrm{M})$. The inhibitory connection of one pair had a briefer IPSP rise time (time to peak, $9.7 \mathrm{msec}$ ) and duration (50\% decay, $39 \mathrm{msec}$ ); the latter IPSP was unaltered by bicuculline but was suppressed by strychnine.

\section{Neuron categories making excitatory connections}

Good biocytin labeling was obtained in both neurons of four neuron pairs that demonstrated a monosynaptic excitatory linkage. In each of these four cases, the presynaptic cell was of the central type; three were of the transient variety, and one was of the tonic type. Three of the four postsynaptic neurons were of the
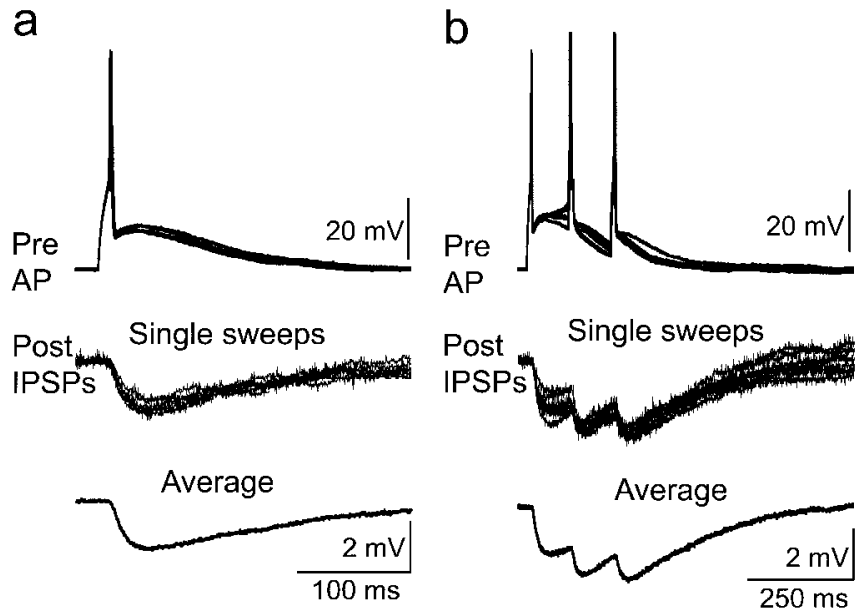

Figure 3. Examples of a monosynaptic inhibitory connection between SG neurons. Top traces, Six superimposed traces recorded from the presynaptic neuron showing action potential (AP) initiated by depolarizing pulses. Holding potential: $-60 \mathrm{mV}$ for presynaptic neuron, -50 $\mathrm{mV}$ for postsynaptic neuron. APs were evoked in the presynaptic cell by single pulses at $0.2 \mathrm{~Hz}$ $(a)$ and by three successive pulses $(b)$. Middle traces, Six traces of recordings from the postsynaptic neuron (Post) showing evoked IPSPs. Bottom traces, Average of 50 successive IPSPs. The latencies from the peak of the presynaptic APs to onset of the IPSPs (2.1 msec) remain relatively constant at $0.2 \mathrm{~Hz}$ ( $a$ ) and for triple repetitions at $100 \mathrm{msec}$ intervals (b).

vertical category, whereas one was a tonic central cell. The latter was excited by a transient central neuron. In two excitatory connections, the presynaptic neuron alone was marked adequately to permit cell type categorization; both of these were transient central neurons. The postsynaptic cell alone was well labeled in two other pairs exhibiting monosynaptic excitation; both were of the vertical type. Responses evoked by DR stimulation varied across this population and often were biphasic (a mixture of EPSCs and IPSCs).

Thus, the excitatory linkages in the present sample appear to represent several varieties. More data are needed to determine whether definite patterns exist; although the examples noted are suggestive of an excitatory projection from certain central to vertical neurons. In contrast to these excitatory interactions, a definitive pattern and more consistency appeared in the inhibitory connections.

\section{Characteristics of an inhibitory connection}

Fourteen pairs of neurons had in common the feature that an action potential in one of the pair evoked a constant latency, relatively slowly developing, and prolonged IPSP in the other neuron. The shared morphological and functional features outlined below suggest that each of these 14 pairs of SG neurons represents the same connection pattern.

In this set, IPSPs were evoked in the receiving cell by single or repeated presynaptic action potentials (Fig. 3). The IPSPs were judged to be monosynaptically generated because of a near constant latency in each pair between the peak of presynaptic action potentials, repeated at $0.1-20 \mathrm{~Hz}$, and onset of the postsynaptic IPSPs. The latency in different pairs varied between 1.2 and 3.3 msec, averaging $2.3 \pm 0.6 \mathrm{msec}(n=14)$. Synaptic transmission was generally reliable, as indicated by a relatively low transmission failure (failure of presynaptic action potentials to evoke an IPSP/number of observations). Failure of transmission never occurred in three pairs, occurred in $<20 \%$ of trials in seven pairs, and appeared in $20-42 \%$ in another four pairs.

At a holding potential of $-50 \mathrm{mV}$, individual IPSPs evoked by 


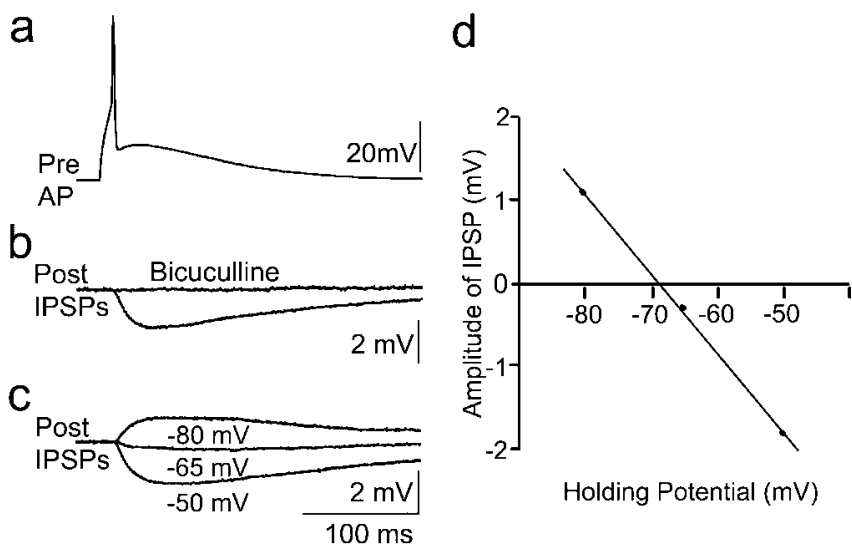

Figure 4. Characterization of an inhibitory connection. Data are from the same pair of cells whose features are also illustrated in Figures 3 and $5 a$. $a$, Action potential (AP) evoked in the presynaptic (Pre) neuron by a depolarizing pulse. $b$, IPSP in the postsynaptic (Post) cell under control conditions and in the presence of bath-applied bicuculline (10 $\mu \mathrm{m})$. c, IPSPs evoked by presynaptic APs at different membrane holding potentials. $d$, Dependence of the IPSPs on postsynaptic membrane potentials. The reversal potential of IPSP was approximately $-70 \mathrm{mV}$. Traces in $a-c$ are averages of 50 trials.

presynaptic action potentials ranged in amplitude from 0.5 to 2.1 $\mathrm{mV}$, averaging $0.96 \pm 0.5 \mathrm{mV}(n=14)$. Figure 3 illustrates fluctuations in the peak amplitude of IPSPs elicited by single or multiple action potentials for a pair that exhibited larger than average IPSP amplitudes. The times to peak for the evoked IPSP for the 14 connected pairs varied from 28.6 to $41.2 \mathrm{msec}$, averaging $34.6 \pm$ $3.6 \mathrm{msec}$, and the half-decay time ranged from 86.6 to $115.5 \mathrm{msec}$, averaging $98.0 \pm 7.5 \mathrm{msec}$. Figure $3 b$ also illustrates that at a holding potential of $-50 \mathrm{mV}$, IPSPs evoked by a succession of action potentials in the presynaptic element summate. The IPSPs initiated by the synaptic connection between neurons of a pair represented outward (hyperpolarizing) current at a holding potential of $-50 \mathrm{mV}$. These IPSPs reversed polarity when the transmembrane potential was increased to near $-70 \mathrm{mV}$ (Fig. $4 c, d$ ). Equilibration between the internal solution and the cell interior was uncertain at the time IPSP reversal potentials were determined (10-20 min after debut of the whole-cell recording); therefore, meaningful comparison with equilibrium values for specific ion species is difficult. The $\mathrm{GABA}_{\mathrm{A}}$ receptor selective antagonist bicuculline $(10 \mu \mathrm{M})$ completely blocked the prolonged evoked IPSPs (Figs. $2 b, 4 b ; n=9$ ). Neither the amplitude nor the duration of such IPSPs was modified by the glycine receptor antagonist strychnine (data not shown). Taken together, these observations suggest that generation of these SG neuron-to-SG neuron IPSPs is dominated by synaptic opening of $\mathrm{GABA}_{\mathrm{A}}$ receptormediated $\mathrm{Cl}^{-}$channels (Hille, 2001).

\section{Neurons forming the inhibitory connection}

Neurons of the rodent SG can be distinguished and classified by a combination of morphological and functional characteristics (Grudt and Perl, 2002; Grudt et al., 2002). On that basis, the 14 inhibitory linked pairs had common features strongly suggesting that they involved the same neuronal types and synaptic arrangements.

\section{Electrophysiological measures}

The resting membrane potentials (RMPs) of the sending (presynaptic) and receiving (postsynaptic) neurons systematically differed. Presynaptic RMPs were significantly less than those of postsynaptic cells in individual pairs (13 of 14) and when aver- aged for the 14 pairs $(-51.1 \pm 2.9$ compared with $-61.8 \pm 5.8$ $\mathrm{mV}$, respectively). The linked neurons also differed in their action potential discharge patterns to prolonged (1000 msec), graded depolarizing pulses (examples shown in Fig. 5). At moderately superthreshold depolarizations, presynaptic cells uniformly discharged tonically (repetitively at relatively regular intervals; 14 of 14), whereas postsynaptic neurons were equally consistent (14 of 14) in exhibiting a burst of impulses followed by silence during maintained depolarization (Fig. 5) (also see Supplement Table 1, available at www.jneurosci.org).

\section{Neuronal morphology}

Morphological features distinguishing SG cells include soma location, soma size, and dendritic distribution. In all 14 inhibitory linked pairs, the recordings were obtained from neurons separated by $<250 \mu \mathrm{m}$, as judged by the position of the electrode tips and biocytin labeling, in an SG zone bracketing the junction between laminae IIi and IIo. In the four pairs in which both neurons were well delineated by biocytin, the presynaptic (sending) cell had a larger soma and a much more elongated dendritic arbor than the postsynaptic (receiving) neuron (e.g., Fig. 5). This difference was upheld by data from another eight pairs in which only either the presynaptic $(n=4)$ or the postsynaptic $(n=4)$ cell was well stained by biocytin, and the position of the other neuron was marked. Measuring the largest dimension, the mean presynaptic soma diameter was $20.2 \pm 1.4 \mu \mathrm{m}(n=8)$, whereas that of the postsynaptic cells was $14.5 \pm 0.7 \mu \mathrm{m}(n=8)$.

Both presynaptic and postsynaptic cells had principal dendritic expansions oriented rostrocaudally with much smaller arborizations in the mediolateral and dorsoventral directions. Dendrites of individual presynaptic neurons extended from 420.7 to $660.2 \mu \mathrm{m}$ rostrocaudally (mean, $525.4 \pm 110.8 \mu \mathrm{m} ; n=8$ ). In contrast, dendritic arbors of the postsynaptic cells were considerably shorter, ranging from 180.3 to $286.8 \mu \mathrm{m}$ (mean, $213.6 \pm$ $43.2 \mu \mathrm{m} ; n=8$ ). This difference was consistent, appearing in all instances in which both cells were well labeled by biocytin $(n=$ $4)$. When only one cell of each pair was well stained, dendritic extension of a presynaptic cell was always large $(450.6-720.8 \mu \mathrm{m}$; $n=4)$, whereas that of postsynaptic cells regularly was much smaller (168.3-266.4 $\mu \mathrm{m} ; n=4$ ) (also see Supplemental Table 1, available at www.jneurosci.org).

Most neurites emanating from the somata of both presynaptic and postsynaptic neurons generally had the tapering features of dendrites. A thin process, of apparently uniform diameter originating from the soma or a proximal dendrite, was also often present and presumed to be the axon of the cell. In the case of the presynaptic neuron of a connected pair, axon-like processes passed near some of the intertwined dendritc processes of the closely positioned neurons; however, morphological definition of synaptic sites between presynaptic and postsynaptic processes in such material, at the best, is uncertain. In general, the axon-like process of the presynaptic neuron branched and was distributed partially within the dendritic field of the parent neuron and was lost in that region, presumably because of pruning in the process of preparing the tissue section. Axon-like neurites of postsynaptic cells usually were lost at the edges of the tissue, often afterward giving rise to a branch passing deep to the soma in lamina IIi.

\section{Classification}

Together, the physiological and morphological features of the presynaptic and postsynaptic neurons matched closely those of two rodent SG neuron types identified by Grudt and Perl (2002). The presynaptic cells regularly exhibited the relatively low RMPs, 
the tonic discharge pattern, the soma location, soma size, and the exceptionally extensive rostrocaudal dendritic spread characteristic of the SG islet cell type. On the other hand, the postsynaptic neurons consistently had higher RMPs than the presynaptic neurons, a transient pattern of action potential generation, lack of an $\mathrm{A}$ current $\left(I_{\mathrm{A}}\right)$, soma size, soma location, and dendritic spread typical for transient (non- $I_{\mathrm{A}}$ ) central-type neurons.

Primary afferent input

Volleys of impulses in primary afferent fibers, generated by stimulation of the segmental DR with brief electrical pulses, evoked EPSPs (or EPSCs) in both neurons of eight inhibitory connected pairs and in one cell each of two other pairs. The latency of the evoked responses for any given pair was regularly (eight of eight) shorter by 3-12.3 msec for the presynaptic than for the postsynaptic cell (Fig. 6). On the basis of the stability of the response latency on repeated trials (e.g., Fig. $6 a$ ), the DRevoked responses were judged to be monosynaptic. Presuming a monosynaptic linkage, the estimated conduction velocity (CV) of the DR afferent fibers evoking the first phase of these responses ranged from 0.23 to $0.49 \mathrm{~m} / \mathrm{sec}$. This $\mathrm{CV}$ range is in keeping with rodent $\mathrm{C}$-fibers at $22-25^{\circ} \mathrm{C}$ (Lynn and Carpenter, 1982; Lawson et al., 1997). The DR CV calculated for the response of the postsynaptic cell was always (eight of eight) slower than that for the presynaptic neuron. Furthermore, consistent with the latency and CV differences, the threshold stimulus intensity for the DR-evoked response in presynaptic neurons was systematically (eight of eight) and on average less (mean, 35\% less; $p<$ 0.05 ) than for postsynaptic cells (Fig. $6 a$ ).

The DR-evoked EPSPs and EPSCs, although varying from one pair to another, often were substantial in amplitude, in some instances exceeding $5 \mathrm{mV}$ (or in voltage clamp, >100 pA). They were particularly large in the presynaptic neuron. On occasion, the DR response initiated an action potential in the presynaptic neuron, which in turn triggered an IPSP in the postsynaptic cell (Fig. 6b), documenting functional effectiveness of the pathway.

\section{Discussion}

These observations provide novel insight on features of the functional organization of the spinal substantia gelatinosa. First, synaptic connections between intrinsic neurons of the SG are shown to be highly selective and particular. This follows from the observations that many randomly selected pairs of neurons ( $90 \%)$ do not show evidence of immediate synaptic interaction. Thus, the SG is not a broadly interconnected region. At the same time, synaptic connections between specific SG neurons are demonstrable.

Second, we document a particular inhibitory connection between two kinds of SG neurons that is related to primary afferent
C-fiber input. Our interpretation of this linkage is shown in Figure 7 . Both neurons of this circuit are located in the midportion of the SG near the interface between laminae IIo and IIi. The presynaptic element of the connection fits the characteristics of the islet type of SG neurons. These islet cells send a monosynaptic inhibitory projection to a nearby neuron whose features match those of the transient type of central neuron. The finding that both the presynaptic islet cell and postsynaptic central cell receive monosynaptic connections from different segments of the primary afferent C-fiber spectrum is significant. This circuit implies that afferent input from one part of the C-fiber spectrum, through excitation of islet cells, inhibits neurons receiving excitatory input from a different subset of primary afferent C-fibers. The inhibitory connection between the islet cell and the central cell is bicuculline-sensitive, suggesting probable $\mathrm{GABA}_{\mathrm{A}}$ mediation (Narikawa et al., 2000). Because the excitatory connection between the afferent $\mathrm{C}$-fibers and both the islet and central cells was reversibly blocked by the AMPA receptor antagonist CNQX, the excitatory primary afferent input is apparently mediated by activation of glutamate AMPA receptors. The $\mathrm{C}$ primary afferent 
a1

DR stimulation ( $4.2 \mathrm{~V})$

(voltage clamp records)

\section{$b_{1}$}

DR stimulation (6 V)

(current clamp records)
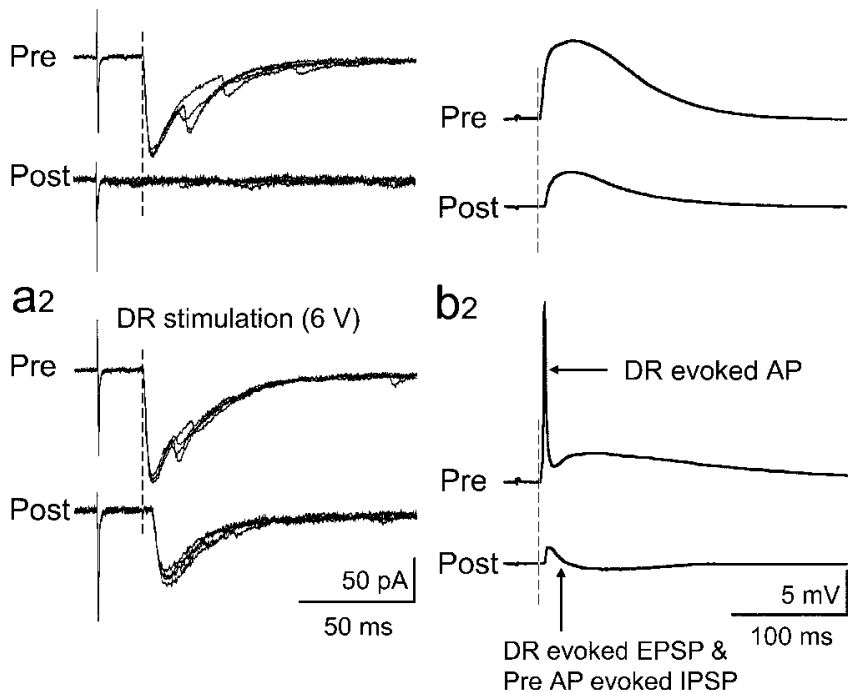

Figure 6. Responses evoked by graded dorsal root stimulation in pairs of inhibitory connected SG neurons. Pre, Presynaptic neuron; Post, postsynaptic neuron. Data are from the same pair of cells as for Figures 3-5a. a, Voltage-clamp recordings (holding potential, $-60 \mathrm{mV}$ ) of individual responses elicited by DR stimulation. $a 1$, Responses (EPSC) appeared in only the presynaptic cell at a relatively weak DR stimulus intensity $(4.2 \mathrm{~V}, 0.5 \mathrm{msec}, 0.1 \mathrm{~Hz})$. a2, Increasing the DR stimulus ( $6 \mathrm{~V}, 0.5 \mathrm{msec}, 0.1 \mathrm{~Hz}$ ) also evoked an EPSC in the postsynaptic cell. Four trials were superimposed in $a 1$ and $a 2$. $b$, Current-clamp recordings (holding potentials: presynaptic, $-60 \mathrm{mV}$; postsynaptic, $-50 \mathrm{mV}) .61$, EPSPs evoked by dorsal root stimulation $(6 \mathrm{~V}, 0.5$ $\mathrm{msec}, 0.1 \mathrm{~Hz}$ ). Note that the latency for EPSP in the postsynaptic cell is longer than that for the presynaptic neuron. $b 2$, The holding potential of the presynaptic neuron was changed from -60 to $-50 \mathrm{mV}$, and at the intensity of DR stimulation for $b 1$, an action potential (AP) was initiated on the rising phase of the DR evoked EPSP. This AP leads to an IPSP at the time of the rising phase of the DR evoked EPSP in the postsynaptic neuron, suppressing most of the latter. Traces in $b 1$ and $b 2$ are averages of 20 trials.

input to an islet cell can be potent enough to initiate action potentials, which, in turn, trigger the inhibitory projection to the postsynaptic central neuron.

The terminology for SG neurons deserves comment. We use the term islet cell to designate a neuron in the mid SG of young, free-ranging hamsters and young adult rats that has a notably elongated (typically $>400 \mu \mathrm{m}$ ) and relatively dense dendritic arborization in the rostrocaudal direction (Grudt and Perl, 2002). That terminology conforms to the early description of similar neurons in the cat SG (Gobel, 1976). Such elongated lamina II neurons are reported to be immunoreactive for GABA (Todd and McKenzie, 1989; Todd and Spike, 1993). We call a similarly oriented cell with a substantially smaller dendritic spread, typically half of the usual islet cell expansion, a central neuron after the description by Ramon y Cajal (1909). Central neurons not only have smaller soma but also a less elongated and less arborized dendritic tree than islet neurons. Todd and McKenzie (1989) and Todd and Spike (1993) have called these smaller neurons "small islet" cells and note that some are not immunoreactive for GABA. Previous studies have suggested that the large islet cells of the SG are inhibitory interneurons (Gobel, 1978; Todd and McKenzie, 1989). Our observations and conclusions are consistent with and extend that concept to a particular pathway.

The in vitro nature of the present experiments does not provide direct information on the afferent modalities represented by

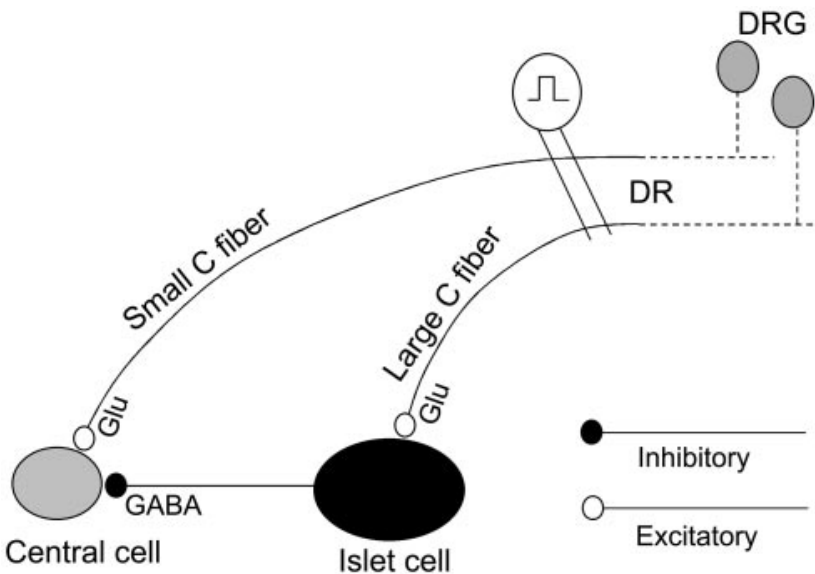

Figure 7. Schematic summarizing the (-fiber-related inhibitory circuit between islet and transient central SG neurons. The presynaptic islet cell has a GABA (bicuculline-sensitive) projection to the transient central neuron. The islet and the transient central neurons receive monosynaptic input from different primary afferent C-fibers via a glutamate AMPA receptormediated connection. The input to the presynaptic islet cell is from larger-diameter, more rapidly conducting C-fibers than those projecting to the postsynaptic transient central neurons. The latter is consistent with the concept that the input to the islet neurons is from C-fibers signaling different (innocuous?) events than those exciting the transient central cells (noxious?). DRG, Dorsal root ganglion.

the dorsal root projection to the islet and central cells; however, there are clues for speculation. In several mammalian species, including rat, the primary afferent C-fiber spectrum includes subsets distinguished by different conduction velocities and afferent properties (Douglas and Ritchie, 1957; Douglas et al., 1960; Lawson et al., 1997; Fang et al., 2002). For nerves supplying hairy skin in a given animal, the more rapidly conducting $C$ population typically is composed of fibers from low-threshold mechanoreceptors or cooling thermoreceptors. In contrast, the more slowly conducting $\mathrm{C}$-fiber class is dominated by heat-sensitive and other types of nociceptors. The consistently lower threshold and shorter latency of DR-evoked EPSPs for the presynaptic islet cell than for the postsynaptic central neuron suggests that the DR excitation of the islet neuron is by relatively larger-diameter, more rapidly conducting $\mathrm{C}$ primary afferents. These considerations raise the possibility that the peripheral $\mathrm{C}$ afferent projections to the presynaptic islet neuron are innocuous mechanoreceptive (or thermoreceptive) fibers, whereas those to the postsynaptic central cell are nociceptive. Therefore, the circuit could represent innocuous impulses suppressing nociceptive input.

The fact that the inhibitory linkage between islet and transient central cells was noted repeatedly is suggestive of the repetition of a neural module in which given types of neurons play functionally equivalent parts. A modular pattern for primary afferent projections in the SG was suggested by Réthelyi (1981) on the basis of Golgi studies. The appearance of apparently identical arrangements in which a particular type of neuron is presynaptic to another specific kind of cell, along with selectively different primary afferent connections to the two elements, can be rationalized by the concept of repetition of a particular circuit pattern. Although one can speculate about the possible significance of this arrangement, eventually fitting it into the functional organization of the spinal superficial dorsal horn requires additional information. In particular, it will become important to determine the axonic projections of the postsynaptic transient central cells. A repeating module of neuronal connectivity associated with dif- 
ferent parts of the C-fiber primary afferent spectrum conceivably could be related to inputs from somatopically different areas. Another possibility is that somehow this modular pathway reflects interaction between different afferent modalities. At the least, our observations indicate that the spinal substantia gelatinosa needs to be considered as a neural complex composed in part of explicit circuits.

Finally, past attention to interactions between inputs involving afferent C-fibers has generally focused on modulation of pain-related activity by impulses in myelinated afferent fibers subserving innocuous mechanoreceptive modalities (Melzack and Wall, 1965; Willis and Coggeshall, 1978; Willis, 1985). The present observations indicate that the SG contains neural circuitry associated with modulation of C-fiber afferent activity by input from other unmyelinated fibers, a point that needs to be integrated into concepts on functional organization of the spinal dorsal horn.

\section{References}

Beal JA, Nandi KN, Knight DS (1989) Characterization of long ascending tract projection neurons and non-tract neurons in the superficial dorsal horn (SDH). In: Processing of sensory information in the superficial dorsal horn of the spinal cord (Cervero F, Bennett GJ, Headley PM, eds), pp 181-197. New York: Plenum.

Budai D (2000) Neurotransmitters and receptors in the dorsal horn of the spinal cord. Acta Biol Szegediensis 44:21-38.

Cervero F, Iggo A (1980) The substantia gelatinosa of the spinal cord: a critical review. Brain 103:717-772.

Douglas WW, Ritchie JM (1957) Non-medullated fibres in the saphenous nerve which signal touch. J Physiol (Lond) 139:385-399.

Douglas WW, Ritchie JM, Straub RW (1960) The role of non-myelinated fibres in signalling cooling of the skin. J Physiol (Lond) 150:266-283.

Fang X, Djouhri L, Black JA, Dib-Hajj SD, Waxman SG, Lawson SN (2002) The presence and role of the tetrodotoxin-resistant sodium channel Nav1.9 (NaN) in nociceptive primary afferent neurons. J Neurosci 22:7425-7433.

Gobel S (1975) Golgi studies of the substantia gelatinosa neurons in the spinal trigeminal nucleus. J Comp Neurol 162:397-416.

Gobel S (1976) Principles of organization in the substantia gelatinosa layer of the spinal trigeminal nucleus. In: Advances in pain research and therapy (Bonica JJ, Albe-Fessard D, eds), pp 165-170. New York: Raven.

Gobel S (1978) Golgi studies of the neurons in layer II of the dorsal horn of the medulla (trigeminal nucleus caudalis). J Comp Neurol 180:395-414.

Gobel S, Falls WM, Bennett GJ, Abdelmoumene M, Hayashi H, Humphrey E (1980) An EM analysis of the synaptic connections of horseradish peroxidase-filled stalked cells and islet cells in the substantia gelatinosa of adult cat spinal cord. J Comp Neurol 194:781-807.

Grudt TJ, Perl ER (2002) Correlations between neuronal morphology and electrophysiological features in the rodent superficial dorsal horn. J Physiol (Lond) 540:189-207.

Grudt TJ, van den Pol AN, Perl ER (2002) Hypocretin-2 (orexin-B) modulation of superficial dorsal horn activity in rat. J Physiol (Lond) 538:517-525.
Hille B (2001) Ion channels of excitable membranes. Sunderland, MA: Sinauer.

Institute of Laboratory Animal Resources, Commission on Life Sciences (1996) Guide for the care and use of laboratory animals. Washington, DC: National Academy.

Kumazawa T, Perl ER (1978) Excitation of marginal and substantia gelatinosa neurons in the primate spinal cord: indications of their place in dorsal horn functional organization. J Comp Neurol 177:417-434.

Lawson SN, Crepps BA, Perl ER (1997) Relationship of substance P to afferent characteristics of dorsal root ganglion neurons in guinea pig. J Physiol (Lond) 505:177-191.

Li J, Perl ER (1994) Adenosine inhibition of synaptic transmission in the substantia gelatinosa. J Neurophysiol 72:1611-1621.

Light AR (1992) The initial processing of pain and its descending control: spinal and trigeminal systems. Basel: Karger.

Light AR, Trevino DL, Perl ER (1979) Morphological features of functionally defined neurons in the marginal zone and substantia gelatinosa of the spinal dorsal horn. J Comp Neurol 186:151-171.

Lynn B, Carpenter SE (1982) Primary afferent units from the hairy skin of the rat hind limb. Brain Res 238:29-43.

Melzack R, Wall PD (1965) Pain mechanisms: a new theory. Science 150:971-979.

Narikawa K, Furue H, Kumamoto E, Yoshimura M (2000) In vivo patchclamp analysis of IPSCs evoked in rat substantia gelatinosa neurons by cutaneous mechanical stimulation. J Neurophysiol 84:2171-2174.

Pearson AA (1952) Role of gelatinous substance of spinal cord in conduction of pain. Arch Neurol Psychiatry 68:515-529.

Perl ER (1984) Pain and nociception. In: Handbook of physiology: the nervous system, Vol 3 (Darian-Smith, ed), pp 915-975. Bethesda, MD: American Physiological Society.

Ramon y Cajal S (1909) Histologie du systéme nerveux de l'homme et des vertébrés, Vol 1. Paris: Maloine.

Réthelyi M (1981) The modular construction of the neuropil in the substantia gelatinosa of the cat's spinal cord: a computer aided analysis of Golgi specimens. Acta Morphol Acad Sci Hung 29:1-18.

Réthelyi M, Szentágothai J (1969) The large synaptic complexes of the substantia gelatinosa. Exp Brain Res 7:258-274.

Sugiura Y, Lee CL, Perl ER (1986) Central projections of identified, unmyelinated (C) afferent fibers innervating mammalian skin. Science 234:358-361.

Szentágothai J (1964) Neuronal and synaptic arrangement in the substantia gelatinosa Rolandi. J Comp Neurol 122:219-239.

Todd AJ, McKenzie J (1989) GABA-immunoreactive neurons in the dorsal horn of the rat spinal cord. Neuroscience 31:799-806.

Todd AJ, Spike RC (1993) The localization of classical transmitters and neuropeptides within neurons in laminae I-III of the mammalian spinal dorsal horn. Prog Neurobiol 41:609-645.

Willis WD (1985) The pain system: the neural basis of nociceptive transmission in the mammalian nervous system. Basel: Karger.

Willis WD, Coggeshall RE (1978) Sensory mechanisms of the spinal cord. New York: Plenum.

Yoshimura M, Jessell TM (1989) Primary afferent-evoked synaptic responses and slow potential generation in rat substantia gelatinosa neurons in vitro. J Neurophysiol 62:96-108.

Yoshimura M, Nishi S (1993) Blind patch-clamp recordings from substantia gelatinosa neuron in adult rat spinal cord slices: pharmacological properties of synaptic currents. Neuroscience 53:519-526. 